\title{
Approach to Neurodegenerative Disease in Children: A Short Review
}

\author{
Shubhankar Mishra* and Ajit Prasad Mishra
}

Department of Neurology, SCB Medical College, India

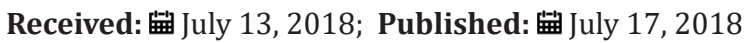

*Corresponding author: Shubhankar Mishra, Department of Neurology, SCB Medical College, India

\begin{abstract}
Neurodegenerative disorders of childhood are complicated diseases with wide range of systematic involvement. These diseases often pose great challenge to clinicians in terms of diagnosis and management. The purpose of this article is to outline a systematic approach to a child presenting with suspected neurodevelopmental regression. Many inherited metabolic disorders present with neural regression. The clinical approach depends upon the age of presentation, site of involvement in brain. Sound clinical knowledge and better approach leads to early diagnosis, better management and above all genetic counselling. As the medical science is in the track of rapid progression several treatment modalities are in the pipeline for neurodegenerative syndromes, early diagnosis and referral to higher centres can bring a better future to the child.
\end{abstract}

Keywords: Neurodegenerative Diseases; Hepatomegaly; White Matter; Grey Matter

\section{Introduction}

Neurodegenerative disorders of childhood include large, heterogeneous group of diseases that result from specific genetic and biochemical defects, chronic viral infections, and varied unknown causes. The hallmark of a neurodegenerative disease is regression and progressive deterioration of neurologic function with loss of speech, vision, hearing, or locomotion, often associated with seizures, feeding difficulties, and impairment of intellect [1]. The acquisition of new developmental milestones does not exclude the existence of a degenerative disorder. Most degenerative CNS disorders can be divided clinically into three groups: graymatter diseases, white-matter diseases, and system diseases [1,2].

\section{Classification}

\section{Approach to child with marked regression:}

Table 1: Area of brain involvement.

\begin{tabular}{|c|c|c|c|}
\hline $\begin{array}{l}\text { Areas of brain } \\
\text { involvement [3] }\end{array}$ & Structural changes & Clinical features & Disease/syndrome \\
\hline Gray-matter & $\begin{array}{l}\text {-lobar gray matter } \\
\text {-the basal } \\
\text { Ganglia and cerebellar nuclei } \\
\text {-ganglion cells of the retina }\end{array}$ & $\begin{array}{c}\text { early onset dementia, progressive loss of } \\
\text { Cognitive abilities, myoclonic seizures } \\
\text {-extrapyramidal } \\
\text { And cerebellar signs, such as ataxia } \\
\text {-pigmentary degeneration } \\
\text { Of the retina }\end{array}$ & $\begin{array}{c}\text { GM1 gangliosidosis } \\
\text { Sandhoffs' disease (GM2 gangliosidosis) } \\
\text { NiemannePick C and related disorders } \\
\text { Sialidosis } \\
\text { Mucopolysaccharidosis (MPS) } \\
\text { Gaucher type III }\end{array}$ \\
\hline White matter & $\begin{array}{l}\text { Abnormal myelin that breaks down } \\
\text { rapidly. These "dysmyelinating" } \\
\text { disorders are called } \\
\text { Leukodystrophies }\end{array}$ & $\begin{array}{c}\text {-the earliest sign spasticity. } \\
\text {-Dementia and seizures } \\
\text { Can occur, but later } \\
\text {-Extrapyramidal signs } \\
\text { Rare, but ataxia due to cerebellar pathway } \\
\text { involvement } \\
\text {-optic atrophy is the most characteristic } \\
\text { ocular change } \\
\text { - cortical blindness }\end{array}$ & $\begin{array}{c}\text { Metachromatic Leukodystrophy (MLD) } \\
\text { Adrenoleukodystrophy (ALD) } \\
\text { Krabbe disease } \\
\text { Alexander disease } \\
\text { Canavan disease } \\
\text { Mitochondrial disorders }\end{array}$ \\
\hline
\end{tabular}




\begin{tabular}{|l|c|c|c|}
\hline System & $\begin{array}{c}\text { Own signs and symptoms, } \\
\text { Depending on the particular neural } \\
\text { Pathways involved }\end{array}$ & $\begin{array}{c}\text { Seizures, stereotypic behaviour, } \\
\text { irritability, } \\
\text { And insomnia, gross delay in milestones }\end{array}$ & Rett syndrome \\
\hline
\end{tabular}

a) History: In a patient with developmental regression the history is very vital. Clues for diagnosis lie in the history. First step would be to ascertain the age of onset of regression and the acquisition of various milestones prior to that. Was the child completely normal before regression or was there a concern Table 2: Approach according to age of presentation [4].

\begin{tabular}{|c|c|c|}
\hline Age group & Disease & Hard clinical features \\
\hline \multirow{8}{*}{$<2$ yr with hepatomegaly } & Fructose intolerance & $\begin{array}{l}\text { Vomiting, hypoglycemia, poor feeding, failure to } \\
\text { thrive (when given fructose) }\end{array}$ \\
\hline & Galactosemia & $\begin{array}{l}\text { Lethargy, hypotonia, icterus, cataract, } \\
\text { hypoglycemia (when given lactose) }\end{array}$ \\
\hline & Glycogenosis (glycogen storage disease) & Hypoglycemia, cardiomegaly \\
\hline & Mucopolysaccharidosis types I and II & Coarse facies, stiff joints \\
\hline & Tay-Sachs disease & $\begin{array}{l}\text { Seizures, cherry-red macula, edema, coarse } \\
\text { facies }\end{array}$ \\
\hline & Gaucher disease (neuronopathic form) & Extensor posturing, irritability \\
\hline & Carbohydrate-deficient glycoprotein syndromes & Dysmyelination, cerebellar hypoplasia \\
\hline & Zellweger syndrome & Hypotonia, high forehead, flat facies \\
\hline \multirow{8}{*}{$<2$ yr without hepatomegaly } & Krabbe disease & $\begin{array}{l}\text { Irritability, extensor posturing, optic atrophy, } \\
\text { and blindness }\end{array}$ \\
\hline & Rett syndrome & $\begin{array}{c}\text { Girls with deceleration of head growth, loss of } \\
\text { hand skills, hand wringing, impaired language } \\
\text { skills, gait apraxia }\end{array}$ \\
\hline & Maple syrup urine disease & Poor feeding, tremors, myoclonus, opisthotonos \\
\hline & Phenylketonuria & Light pigmentation, eczema, seizures \\
\hline & Menkes kinky hair disease & Hypertonia, irritability, seizures, abnormal hair \\
\hline & $\begin{array}{l}\text { Subacute necrotizing encephalopathy of Leigh } \\
\text { disease }\end{array}$ & White matter disease \\
\hline & Canavan disease & White matter disease, macrocephaly \\
\hline & $\begin{array}{l}\text { Neurodegeneration with brain iron } \\
\text { accumulation disease }\end{array}$ & White matter disease, movement disorder \\
\hline \multirow{10}{*}{$2-5$ yrs } & Niemann Pick disease types III and IV & Hepatosplenomegaly, gait difficulty \\
\hline & Wilson disease Liver disease & $\begin{array}{l}\text { Kayser-Fleischer ring; deterioration of cognition } \\
\text { is late }\end{array}$ \\
\hline & Gangliosidosis type II & Gray matter disease \\
\hline & Neuronal ceroid lipofuscinosis & Gray matter disease \\
\hline & Mitochondrial encephalopathies & $\begin{array}{l}\text { myoclonic epilepsy with ragged red fibers } \\
\text { [MERRF]) }\end{array}$ \\
\hline & Ataxia telengactasia & Basal ganglia disease \\
\hline & Huntington disease (chorea) & Basal ganglia disease \\
\hline & $\begin{array}{l}\text { Neurodegeneration with brain iron } \\
\text { accumulation syndrome }\end{array}$ & Basal ganglia disease \\
\hline & Adrenoleukodystrophy & $\begin{array}{l}\text { White matter disease, behavior problems, } \\
\text { deteriorating school performance, quadriparesis }\end{array}$ \\
\hline & Metachromatic leukodystrophy & White matter disease \\
\hline
\end{tabular}

regarding development even prior to regression? Did the child attained milestones before losing it? Which milestones the child lost? In autism and other pervasive developmental disorders regression of language skills is noted first followed by loss of social skills (Tables 1-5). 


\begin{tabular}{|l|c|c|}
\hline \multirow{4}{*}{5} & Multiple sclerosis & White matter disease \\
\cline { 2 - 3 } & $\begin{array}{c}\text { Neuronal ceroid lipofuscinosis, juvenile and } \\
\text { adult (Spielmeyer-Vogt and Kufs disease) }\end{array}$ & $\begin{array}{c}\text { White matter disease, focal neurologic } \\
\text { symptoms }\end{array}$ \\
\cline { 2 - 3 } & Schilder disease & $\begin{array}{c}\text { Peripheral neuropathy, ataxia, retinitis } \\
\text { pigmentosa }\end{array}$ \\
\cline { 2 - 3 } & Refsum disease & $\begin{array}{c}\text { Cherry-red macula, myoclonus, ataxia, coarse } \\
\text { facies }\end{array}$ \\
\cline { 2 - 3 } & Sialidosis II, juvenile form & $\begin{array}{c}\text { Diffuse encephalopathy, myoclonus; may occur } \\
\text { years after measles }\end{array}$ \\
\cline { 2 - 3 } & Subacute sclerosing panencephalitis &
\end{tabular}

Table 3: Treatable conditions with neural regression [5] loss of social skills.

\begin{tabular}{|c|}
\hline Biotinidase deficiency \\
\hline Wilson disease \\
\hline NiemannePick C \\
\hline Neurotransmitter disorder e.g. Segawa, tyrosine hydroxylase deficiency \\
\hline Cerebral folate disorder \\
\hline Glucose transporter disorder \\
\hline Biotin responsive basal ganglia disease \\
\hline Pyruvate dehydrogenase deficiency \\
\hline Creatine disorders \\
\hline
\end{tabular}

Table 4: Specific pointers in history.

\begin{tabular}{|c|c|}
\hline History & Points to be asked \\
\hline \multirow{8}{*}{ Present history } & Seizures \\
\hline & Cognitive impairment \& deterioration in school performance \\
\hline & Gait disturbances: spasticity, ataxia, bradykinesia, dystonia \\
\hline & Personality and behavioural change \\
\hline & Headache and projectile vomiting \\
\hline & Exaggerated startle response \\
\hline & Faltering growth \\
\hline & Feeding difficulties \\
\hline \multirow{8}{*}{ Birth history } & Decrease intrauterine movements \\
\hline & Prematurity \\
\hline & Very low birth weight \\
\hline & Birth asphyxia \\
\hline & Neonatal jaundice \\
\hline & Birth trauma \\
\hline & Neonatal hypoglycaemia \\
\hline & Neonatal seizures \\
\hline \multicolumn{2}{|c|}{ Developmental history } \\
\hline \multirow{3}{*}{ Family history } & Consanguinity \\
\hline & Early onset deaths \\
\hline & Previous affected siblings \\
\hline
\end{tabular}


Table 5: Individual disease and clues for diagnosis.

\begin{tabular}{|c|c|c|c|}
\hline Disease & Clinical clue & Investigation & Treatment \\
\hline Fructose intolerance [6] & $\begin{array}{c}\text { Vomiting, Hypotonia, FTT } \\
\text { Neuropathy, Hepatomegaly } \\
\text { More symptoms with fructose diet }\end{array}$ & $\begin{array}{l}\text { a fructose tolerance test gene } \\
\text { sequencing }\end{array}$ & $\begin{array}{l}\text { Exclusion of fructose from } \\
\text { diet }\end{array}$ \\
\hline Galactosemia [7] & $\begin{array}{c}\text { Lethargy, hypoglycemia hypotonia, } \\
\text { jaundice, cataract, seizure, ataxia, } \\
\text { hepatomegaly, FTT }\end{array}$ & $\begin{array}{l}\text { Beutler's test and the Hill test NBS, } \\
\text { CVS }\end{array}$ & $\begin{array}{l}\text { eliminating lactose and } \\
\text { galactose from the diet. }\end{array}$ \\
\hline $\begin{array}{l}\text { Glycogenosis (glycogen storage } \\
\text { disease) [8] }\end{array}$ & $\begin{array}{l}\text { Hypoglycemia, cardiomegaly } \\
\text { FTT, muscle disease hepatomegaly }\end{array}$ & $\begin{array}{l}\text { Liver biopsy } \\
\text { Genetic testing }\end{array}$ & $\begin{array}{l}\text { Enzyme replacement therapy } \\
\text { for GSD-II } \\
\text { Symptomatic management }\end{array}$ \\
\hline $\begin{array}{l}\text { Mucopolysaccharidosis types I and } \\
\text { II }[9,10]\end{array}$ & $\begin{array}{l}\text { Hurler (type-I): coarse facies, vertebral } \\
\text { anomaly, cloudy cornea } \\
\text { Hunter (type-II): coarse facies, clear } \\
\text { cornea, bone changes }\end{array}$ & $\begin{array}{l}\text { Clinical features } \\
\text { Enzyme assay }\end{array}$ & $\begin{array}{c}\text { Enzyme replacement } \\
\text { Bone marrow transplant }\end{array}$ \\
\hline Tay-Sachs disease [11] & $\begin{array}{l}\text { cognitive and motor skill } \\
\text { deterioration, dysarthria, } \\
\text { dysphagia, ataxia, and spasticity }\end{array}$ & enzyme assay of hexoaminidase & symptomatic \\
\hline $\begin{array}{l}\text { Gaucher disease (neuronopathic } \\
\text { form) [12] }\end{array}$ & $\begin{array}{l}\text { Hepatosplenomegaly } \\
\text { Pancytopenia, bone pain, seizure, } \\
\text { osteoporosis }\end{array}$ & $\begin{array}{l}\text { Enzyme testing for } \\
\text { glucocerebrosidase }\end{array}$ & Enzyme replacement therapy \\
\hline Zellweger syndrome [13] & $\begin{array}{l}\text { Syndromic facies, hypotonia, seizure, } \\
\text { apnea, cartilage disease }\end{array}$ & elevated very long chain fatty acids & $\begin{array}{l}\text { LCT, Lorenzo oil, diet } \\
\text { restriction, fat soluble } \\
\text { vitamins }\end{array}$ \\
\hline Krabbe disease [14] & $\begin{array}{c}\text { irritability, fevers, limb stiffness, seizures, } \\
\text { feeding difficulties, vomiting, slowing of } \\
\text { mental and motor development }\end{array}$ & $\begin{array}{l}\text { multinucleated globoid cells, nerve } \\
\text { demyelination and degeneration }\end{array}$ & $\begin{array}{l}\text { Bone marrow transplantation, } \\
\text { symptomatic treatment }\end{array}$ \\
\hline Rett syndrome $[15,16]$ & $\begin{array}{l}\text { Cognitive skill loss, seizure, muscle } \\
\text { weakness, autistic behaviour }\end{array}$ & $\begin{array}{l}\text { Clinical features } \\
\text { MECP2 mutation }\end{array}$ & Symptomatic treatment \\
\hline Maple syrup urine disease [17] & $\begin{array}{l}\text { sweet-smelling urine, hallucinations, } \\
\text { anorexia, weight loss, anemia, } \\
\text { diarrhea, vomiting, dehydration }\end{array}$ & plasma amino acid measurement, & Symptomatic, diet control \\
\hline Phenylketonuria [18] & $\begin{array}{c}\text { Hypopigmentation, intellectual disability, } \\
\text { microcephaly, seizures, musty mousy } \\
\text { order of skin }\end{array}$ & $\begin{array}{l}\text { New born screening test Tendem } \\
\text { mass spectrometry }\end{array}$ & $\begin{array}{l}\text { PKU Diet, symptomatic } \\
\text { treatment }\end{array}$ \\
\hline Menkes kinky hair disease [19] & $\begin{array}{c}\text { Developmental delay, irritability, fragile } \\
\text { hypopigmented hair, FTT, Seizure, } \\
\text { osteoporosis }\end{array}$ & $\begin{array}{l}\text { copper and ceruloplasmin } \\
\text { levels, skin biopsy, and optical } \\
\text { microscopic examination of the } \\
\text { hair Urine homovanillic acid/ } \\
\text { vanillylmandelic acid ratio }\end{array}$ & $\begin{array}{c}\text { Copper supplement } \\
\text { Symptomatic treatment }\end{array}$ \\
\hline $\begin{array}{c}\text { Subacute necrotizing } \\
\text { encephalopathy of Leigh disease } \\
{[20]}\end{array}$ & $\begin{array}{c}\text { Hypotonia, ataxia, diarrhea, vomiting, } \\
\text { opthalmoparesis, nystagmus, peripheral } \\
\text { neuropathy }\end{array}$ & Serum lactate raised, genetic testing & $\begin{array}{l}\text { Succinic acid, thiamine, } \\
\text { sodium citrate, bicarbonate } \\
\text { Symptomatic treatment }\end{array}$ \\
\hline Canavan disease [21] & $\begin{array}{l}\text { intellectual disability, feeding difficulties, } \\
\text { abnormal muscle tone, poor head control, } \\
\text { and megalocephaly. Paralysis, blindness, } \\
\text { seizures }\end{array}$ & $\begin{array}{l}\text { high concentration of } \\
\mathrm{N} \text {-acetylaspartic acid (NAA) in the } \\
\text { urine, molecular genetic testing }\end{array}$ & Treatment is symptomatic \\
\hline $\begin{array}{l}\text { Neurodegeneration with brain iron } \\
\text { accumulation disease [22] }\end{array}$ & $\begin{array}{l}\text { Pyramidal, extrapyramidal features, } \\
\text { cognitive decline, optic atrophy, retinal } \\
\text { degeneration, seizures }\end{array}$ & MRI of brain & Symptomatic treatment \\
\hline Niemann Pick type C [23] & $\begin{array}{l}\text { Hepatosplenomegaly, dysarthria, ataxia, } \\
\text { dystonia, seizures }\end{array}$ & Clinical features genetic study & $\begin{array}{c}\text { Symptomatic } \\
\text { In NPC-Miglustat [24] }\end{array}$ \\
\hline Wilson disease Liver disease [25] & $\begin{array}{c}\text { neuropsychiatric features, dystonia, KF } \\
\text { Ring, }\end{array}$ & $\begin{array}{l}\text { Serum ceruloplasmin, urinary } \\
\text { copper }\end{array}$ & $\begin{array}{c}\text { Zinc, penicillamine, } \\
\text { symptomatic treatment }\end{array}$ \\
\hline Gangliosidosis type II [26] & $\begin{array}{c}\text { skeletal abnormalities, seizures, profound } \\
\text { intellectual disability, vision loss, } \\
\text { distinctive facial features }\end{array}$ & Genetic testing & Symptomatic treatment \\
\hline
\end{tabular}




\begin{tabular}{|c|c|c|c|}
\hline Neuronal ceroid lipofuscinosis [27] & $\begin{array}{l}\text { vision loss due to retinal dystrophy, with } \\
\text { seizures, psychological degeneration }\end{array}$ & $\begin{array}{c}\text { Eye test, enzyme assay, skin tissue } \\
\text { sampling }\end{array}$ & $\begin{array}{l}\text { Gene therapy, stem cell } \\
\text { therapy, flupirtine [28], } \\
\text { cystagon [29] }\end{array}$ \\
\hline Ataxia telengactasia [30] & $\begin{array}{l}\text { Ataxia, occulomotor apraxia, } \\
\text { telengiectasia, repeated infections, } \\
\text { multiple malignancy }\end{array}$ & $\begin{array}{l}\text { Raised AFP, low level IgA, IgE, } \\
\text { Chromosomal assay, MRI }\end{array}$ & Symptomatic treatment \\
\hline Huntington disease [31] (chorea) & $\begin{array}{l}\text { poor academic performance, or evident } \\
\text { regression in cognitive and language } \\
\text { skills, chorea, behavioural anomaly, } \\
\text { seizure }\end{array}$ & Genetic testing for CAG repeats & symptomatic \\
\hline Adrenoleukodystrophy [32] & $\begin{array}{l}\text { Musclestiffness, paraparesis, dementia, } \\
\text { progressive neuropathy, autonomic } \\
\text { features, addisons disease }\end{array}$ & New born screening, plasma VLCFA & $\begin{array}{l}\text { gene therapy dietary therapy, } \\
\text { symptomatic management }\end{array}$ \\
\hline Metachromatic leukodystrophy [33] & $\begin{array}{l}\text { muscle wasting and weakness, muscle } \\
\text { rigidity, developmental delays, } \\
\text { progressive loss of vision leading } \\
\text { to blindness, convulsions, impaired } \\
\text { swallowing, paralysis, and dementia. late } \\
\text { infantile form, which is the most common } \\
\text { form of MLD }\end{array}$ & $\begin{array}{c}\text { An ARSA-A enzyme level blood test } \\
\text { with a confirming urinary sulfatide } \\
\text { test is the best biochemical test for } \\
\text { MLD }\end{array}$ & Symptomatic treatment \\
\hline $\begin{array}{c}\text { Subacute sclerosing } \\
\text { panencephalitis (SSPE) [33] }\end{array}$ & $\begin{array}{l}\text { primary measles infection followed } \\
\text { by several asymptomatic years (6- } \\
15 \text { on average), and then gradual, } \\
\text { progressive psychoneurological } \\
\text { deterioration, consisting of personality } \\
\text { change, seizures, myoclonus, } \\
\text { ataxia, photosensitivity, ocular } \\
\text { abnormalities, spasticity, and coma. }\end{array}$ & $\begin{array}{l}\text { periodic activity (Rademecker } \\
\text { complex) in EEG elevated anti- } \\
\text { measles antibody (IgG) in the } \\
\text { serum and cerebrospinal fluid, and } \\
\text { typical histologic findings in brain } \\
\text { biopsy }\end{array}$ & $\begin{array}{l}\text { oral isoprinosine) combined } \\
\text { with intrathecal or } \\
\text { intraventricular interferon } \\
\text { alpha }[34,35]\end{array}$ \\
\hline
\end{tabular}

\section{References}

1. Johnston M (2016) Neurodegenerative disorders of childhood. In: Behrman RE, Kliegman RM, Jenson HB, (Eds.); Nelson textbook of pediatrics $\left(20^{\text {th }}\right.$ edn.).

2. Ellaway C, Christodoulou J (1987) Degenerative central nervous system (CNS) disease: In Workshop and Symposium. Percy 2: 82.

3. Henry M Adam (2001) Degenerative Central Nervous System (CNS) Disease, Pediatrics in Review 22(5): 175-176

4. Sreekantam S, Wassmer E (2013) An approach to developmental regression. Paediatrics and Child Health 23(6): 273-277.

5. Pierre G (2013) Neurodegenerative disorders and metabolic disease. Archives of disease in childhood 98(8): 618-24.

6. Maitre A, Maw A, Ramaswami U, Morley SL (2016) Relapsing Acute Axonal Neuropathy in Hereditary Fructose Intolerance. Pediatric neurology 64: 92-93.

7. Goodin B (2006) An overview of expanded newborn screening for inborn errors of metabolism. Practical Gastroenterology 30(8): 34.

8. Bembi B, Cerini E, Danesino C, Donati MA, Gasperini S, et al. (2008) Management and treatment of glycogenosis type II. Neurology 71(232): S12-S36.

9. Wraith JE, Scarpa M, Beck M, Bodamer OA, De Meirleir L, et al. (2008) Mucopolysaccharidosis type II (Hunter syndrome): A clinical review and recommendations for treatment in the era of enzyme replacement therapy. European journal of paediatrics 167(3): 267-277.

10. Vellodi A, Young EP, Cooper A, Wraith JE, Winchester B, et al. (1997) Bone marrow transplantation for mucopolysaccharidosis type I: Experience of two British centres. Archives of disease in childhood 76(2): 92-99.

11. Desnick RJ, Kaback MM (2001) Tay-Sachs disease. Academic Press, USA.

12. Weinreb NJ, Charrow J, Andersson HC, Kaplan P, Kolodny EH, et al. (2002) Effectiveness of enzyme replacement therapy in 1028 patients with type 1 Gaucher disease after 2 to 5 years of treatment: a report from the Gaucher Registry. The American journal of medicine 113(2):112-119.
13. Brown FR, McAdams AJ, Cummins JW, Konkol R, Singh I, et al. (1982) Cerebro-hepato-renal (Zellweger) syndrome and neonatal adrenoleukodystrophy: Similarities in phenotype and accumulation of very long chain fatty acids. The Johns Hopkins medical journal 151(6): 344-351.

14. Suzuki K, Suzuki Y (1970) Globoid cell leucodystrophy (Krabbe's disease): Deficiency of galactocerebroside $\beta$-galactosidase. Proceedings of the National Academy of Sciences 66(2): 302-309.

15. Hagberg B, Goutières F, Hanefeld F, Rett A, Wilson J (1985) Rett syndrome: criteria for inclusion and exclusion. Brain and Development $7(3): 372-373$.

16. Amir RE, Van den Veyver IB, Wan M, Tran CQ, Francke U, et al. (1999) et.al Rett syndrome is caused by mutations in X-linked MECP2, encoding methyl-CpG-binding protein 2. Nature genetics 23(2): 185.

17. Strauss KA, Puffenberger EG, Morton DH (2006) Maple syrup urine disease.

18. Blau N, van Spronsen FJ, Levy HL (2010) Phenylketonuria. The Lancet 376(9750): 1417-1427.

19. Kaler SG, Holmes CS, Goldstein DS, Tang J, Godwin SC (2008) Neonatal diagnosis and treatment of Menkes disease. New England Journal of Medicine 358(6): 605-614.

20. Pincus JH (1972) Subacute necrotizing encephalomyelopathy (Leigh's disease): A consideration of clinical features and etiology. Developmental Medicine \& Child Neurology 14(1): 87-101.

21. Kumar S, Mattan NS, de Vellis J (2006) Canavan disease: A white matter disorder. Developmental Disabilities Research Reviews 12(2): 157-165.

22. Gregory A, Hayflick SJ (2005) Neurodegeneration with brain iron accumulation. Folia Neuropathologica 43(4): 286-296.

23. Sévin M, Lesca G, Baumann N, Millat G, Lyon-Caen O, et al. (2006) The adult form of Niemann-Pick disease type C. Brain130(1): 120-133.

24. Patterson MC, Vecchio D, Prady H, Abel L, Wraith JE (2007) Miglustat for treatment of Niemann-Pick $\mathrm{C}$ disease: a randomised controlled study. The Lancet Neurology 6(9): 765-772. 
25. Brewer GJ, Yuzbasiyan-Gurkan V (1992) Wilson disease. Medicine 71(3): 139-164.

26. Patel V, Goebel HH, Watanabe I, Zeman W (1974) Studies on G M1gangliosidosis, type II. Acta neuropathologica 30(2): 155-173.

27. Jalanko A, Braulke T (2009) Neuronal ceroid lipofuscinoses. Biochimica et Biophysica Acta (BBA)-Molecular Cell Research 1793(4): 697-709.

28. Dhar S, Bitting RL, Rylova SN (2002) Flupirtine blocks apoptosis in batten patient lymphoblasts and in human postmitotic CLN3- and CLN2 deficient neurons. Annals of Neurology 51(4): 448-466.

29. Levin SW, Baker EH, Zein WM, Zhang Z, Quezado ZM, et al. (2014) Oral cysteamine bitartrate and $\mathrm{N}$-acetylcysteine for patients with infantile neuronal ceroid lipofuscinosis: A pilot study. The Lancet Neurology 13(8): 777-787.

30. Lavin MF (2008) Ataxia-telangiectasia: From a rare disorder to a paradigm for cell signalling and cancer. Nature reviews Molecular cell biology 9(10): 759 .
31. Letort D, Gonzalez-Alegre P (2013) Huntington's disease in children. In Handbook of clinical neurology 113: 1913-1917.

32. Schaumburg HH, Powers JM, Raine CS, Suzuki K, Richardson EP (1975) Adrenoleukodystrophy: A clinical and pathological study of 17 cases. Archives of Neurology 32(9): 577-591.

33. Gieselmann V, Krägeloh-Mann I (2010) Metachromatic leukodystrophy: An update. Neuropediatrics 41(01): 1-6.

34. Nihei K (1997) Subacute sclerosing panencephalitis (SSPE): Nihon rinsho. Japanese journal of clinical medicine 55(4): 908-915.

35. Yalaz K, Anlar B, Oktem F, Aysun S, Ustacelebi S, et al. (1992) Intraventricular interferon and oral inosiplex in the treatment of subacute sclerosing panencephalitis. Neurology 42(3): 488-491.

\section{cc) This work is licensed under Creative}

To Submit Your Article Click Here: Submit Article

DOI: $10.32474 /$ PAPN.2018.01.000121

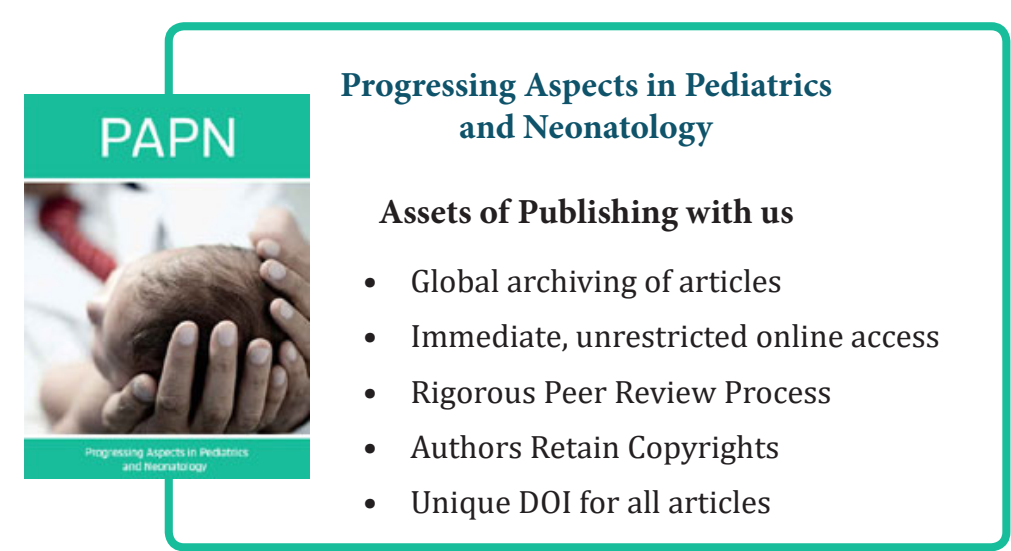

